\title{
BEAM DYNAMICS SIMULATIONS FOR THE GSI HIGH CURRENT INJECTOR WITH THE NEW VERSATILE COMPUTER CODE DYNAMION
}

\author{
J. Klabunde, W. Barth, GSI, Darmstadt, Germany \\ S. Iaramychev, A. Kolomiets, ITEP, Moscow, Russia
}

\section{Abstract}

The multi-particle code DYNAMION was developed for beam dynamics simulations of complex beam transport lines and accelerating structures. The equations of motion are solved by time integration including threedimensional external fields and space charge forces.

Simulations were performed at the different sections of the new high current injector at GSI: LEBT, $36 \mathrm{MHz}$ RFQ, two IH tanks, stripper section, Alvarez tank and beam line with octupole lenses. New features added to DYNAMION for these calculations are described.

After commissioning of the linac, the results of simulations can be compared with beam measurements along the linac. Discrepancies between DYNAMION and other standard simulation codes will be discussed.

\section{INTRODUCTION}

The UNILAC has been upgraded as a high current linac for synchrotron injection and also for physics experiments at low energies. The present layout is shown in Fig. 1. The new prestripper linac consists of a $36 \mathrm{MHz}$ RFQ and two IH-tanks [1]. The injector should provide an increase of beam intensities of more than two orders of magnitude for the heaviest elements. The strong space charge forces at different sections of the linac can deteriorate the beam brilliance to an unacceptable level. Therefore, the computer code DYNAMION has been developed for more realistic modeling the physics of high intensity transport and acceleration of ions.

\section{DESCRIPTION OF DYNAMION}

The multi-particle code DYNAMION has been developed for ion beam dynamics in high current linear accelerators in collaboration between ITEP and GSI [2]. It was written on the base of PROTON created at ITEP about twenty years ago.

DYNAMION solves numerically the general three dimensional equations of charged particle motion in arbitrary external fields by time integration, internal self fields of the ions are taken into account:

$$
\begin{aligned}
& \vec{r}=\vec{r}_{0}+\Delta t \cdot \frac{\vec{v}+\vec{v}_{0}}{2}, \quad \vec{v}=\vec{v}_{0}+\Delta t \cdot \vec{A} \\
& \vec{A}=\frac{1}{\gamma} \cdot \frac{q}{m} \cdot\left(\vec{E}-\frac{\vec{v}_{0}}{c} \cdot\left(\frac{\vec{v}_{0}}{c} \cdot \vec{E}\right)+\left[\frac{\vec{v}_{0}}{c} \times \vec{H}\right]\right) \\
& \vec{E}=\vec{E}_{\text {ext }}+\vec{E}_{\mathrm{int}}, \quad \vec{H}=\vec{H}_{\text {ext }}+\vec{H}_{\mathrm{int}}
\end{aligned}
$$

$\vec{r}, \vec{v}, \vec{A}$ - radius, velocity, acceleration vectors, $q, m-$ charge, mass numbers, $E_{e x t}, H_{e x t}$ - external electrical, magnetic fields, $E_{\text {int }}, H_{\text {int }}$ - internal self fields of ions.

All sections of a modern ion linac can be simulated with high accuracy: RFQ, IH, Alvarez structures, complex transport lines consisting of quadrupoles, bending magnets, solenoids, hexapoles, octupoles, rf cavities et al. External electromagnetic fields can be provided by analytical expressions, field maps calculated by other codes (e.g MAFIA, OPERA) and field measurements. The simultaneous transport or acceleration of beams consisting of different ion species or charge states is also implemented. The space charge forces are calculated by particle-particle interaction, the high number of space charge calculations (e.g. 100 steps per RFQ cell) increases the accuracy of simulations. The results of simulations can be evaluated by the powerful graphic system PAW, available from the CERN program library.

The method chosen in DYNAMION requires large computing time and a large storage system. As an example, to trace 1500 macroparticles through a high current RFQ of 360 cells, DYNAMION needs about 10 hours CPU time, it is a factor of 50 more than required by

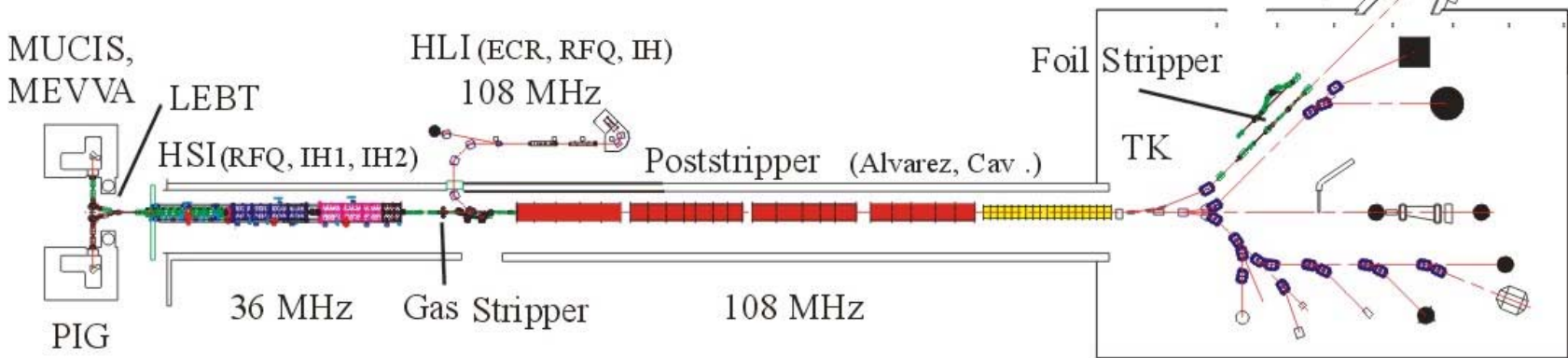

Fig. 1 Scheme of the GSI linear accelerator with the new High Current Injector HSI. 
PARMTEQ. In the following paragraphs some applications of the DYNAMION code will be reported.

\section{HSI RFQ SIMULATIONS}

The new H-type RFQ structure of the HSI accelerates ions with $\mathrm{A} / \mathrm{q}$ ratios up to 65 from 2.2 to $120 \mathrm{keV} / \mathrm{u}$. The expected output beam intensities should be up to $0.25 \cdot \mathrm{A} / \mathrm{q}(\mathrm{emA})$. The profile table of RFQ electrodes was calculated by the PARMTEQ code. With design parameters PARMTEQ calculates a total particles transmission of $95 \%$, the design final energy of $120 \mathrm{keV} / \mathrm{u}$ attain $90 \%$ of injected ions. A later examination by DYNAMION resulted in a considerably lower transmission at the design input current level: $83 \%$ total transmission, $77 \%$ for accelerated ions. The main reason for the discrepancy is the different treatment of space charge calculations. DYNAMION has a higher number of integration steps and space charge calls per RFQ cell. Also an improved treatment of the transition from the unbunched to the bunched beam increases our confidence in the DYNAMION program. After the commissioning of the RFQ section the results of DYNAMION and PARMTEQ simulations can be compared with the measured transmission. The results are shown in Fig. 2. For a more realistic comparison the simulations used a measured transversal input emittance. The discrepancy between PARMTEQ and DYNAMION calculations is evident. The even lower measured transmission can be explained if measured misalignments of electrodes were taken into account in DYNAMION simulations. A realignment of the RFQ is planned.

A further advantage of the DYNAMION code was used for RFQ simulations: the geometry of the electrodes can

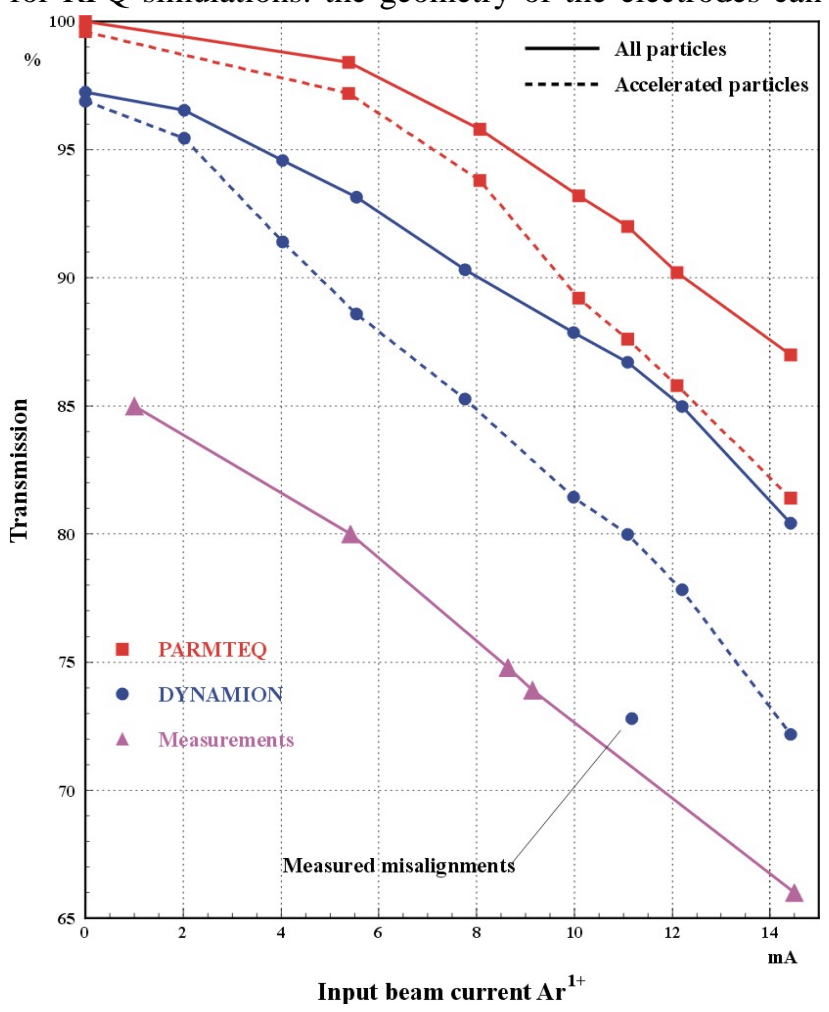

Fig. 2 Transmission through the HSI RFQ. be implemented as fabricated. In Fig. 3 the measured output energy is compared with PARMTEQ and DYNAMION simulations. Surprisingly the measured energy deviates from the expected design energy as calculated by PARMTEQ. An inspection of the machining lists for the electrodes indicated deviations from the design table; especially the end cells were modified. With the real geometry the DYNAMION results agreed to the measurements.

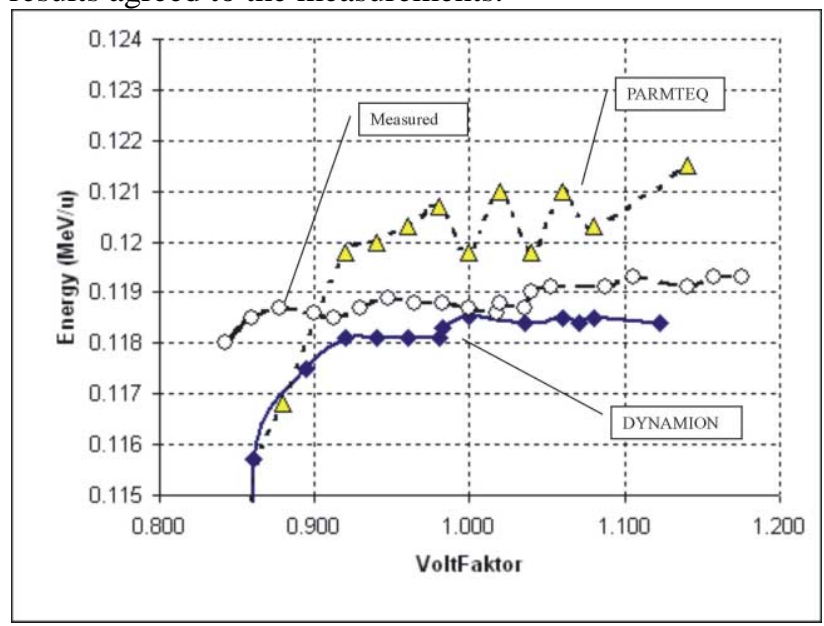

Fig. 3 Output energy of HSI RFQ in dependence of $\mathrm{rf}$ voltage.

\section{DRIFT TUBE LINAC SIMULATIONS}

For beam dynamics of drift tube linacs (DTL), a new subroutine for rf field calculations was implemented. Assuming axial geometry, the Laplace equation is solved by the finite element method. The potential and field components are approximated by series with 30 coefficients:

$$
\begin{aligned}
& U(r, z)=-V\left[\frac{z}{l}+\sum_{n=1}^{\infty} A_{n} \cdot \sin (2 n k z) \cdot I_{0}(2 n k r)\right] \\
& E_{z}(r, z)=-V\left[\frac{1}{l}+\sum_{n=1}^{N} A_{n} \cos (2 n k z) \cdot 2 n k \cdot I_{0}(2 n k r)\right] \\
& E_{r}(r, z)=-V \cdot \sum_{n=1}^{N} A_{n} \sin (2 n k z) \cdot 2 n k \cdot I_{1}(2 n k r) \\
& V=\int_{-l}^{l} E_{z}(0, z) d z, l-\text { half of cell length, } k=\pi / l .
\end{aligned}
$$

The field calculations by DYNAMION were compared with results of the OPERA code. A good agreement of field calculations was achieved up to $90 \%$ of the drift tube aperture. Beam dynamics simulations were carried out for the UNILAC IH and Alvarez structures. In Fig. 4 the longitudinal field distribution on axis for the IH-2 tank is shown. The voltage factors along the tank were obtained by field measurements. 


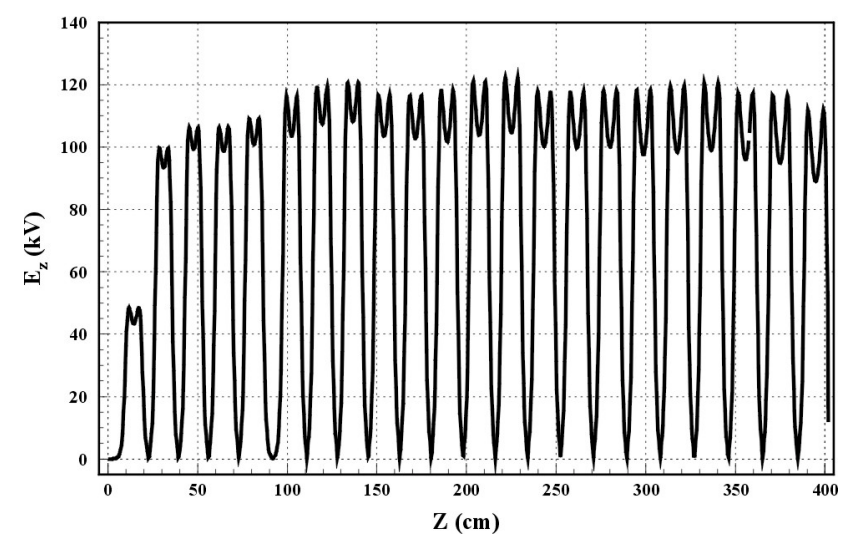

Fig. 4 Longitudinal electrical field of the IH-2 tank calculated by DYNAMION. Voltage factors were obtained from bead pull measurements.

\section{BEAM DYNAMICS AT THE STRIPPER SECTION}

Most severe space charge forces occur after the charge state jump in the gas stripper at $1.4 \mathrm{MeV} / \mathrm{u}$. For optimization of the stripper geometry and the subsequent beam transport section, DYNAMION simulates the multicharge transport after the stripping. A theoretical or measured charge state distribution can be implemented. Space charge forces of all neighboring charge states are taken into account. Beam envelopes, transversal and longitudinal emittances of the reference ion have been calculated. As an example the emittances for different charge states before the analyzing slits are shown (Fig. 5). Due to the space charge forces the charge state separation is not complete, a readjustment of quadrupole fields is necessary.
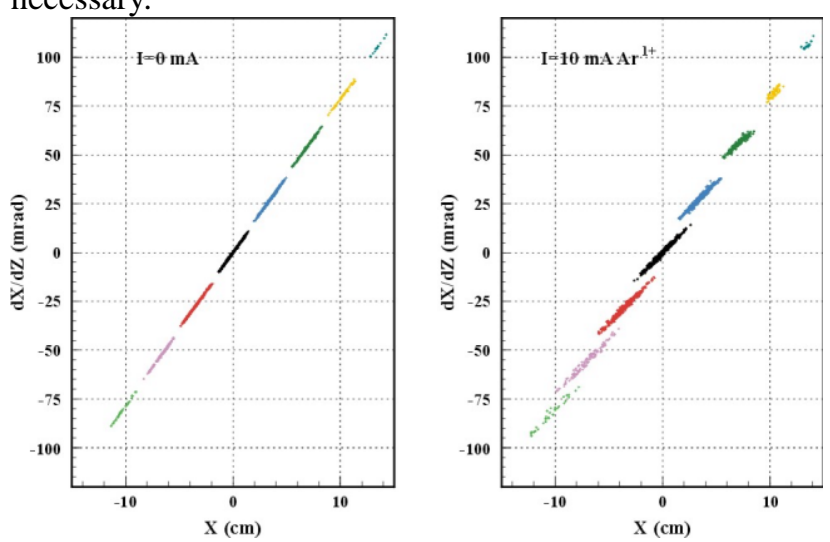

Fig. 5 Phase portraits of the beam in X-plane behind the bending magnet at zero and full current.

\section{UNIFORMITY IMPROVEMENT BY OCTUPOLES}

The spatial uniformity of particle distribution at the target position can be improved by octupole lenses. DYNAMION calculates the beam dynamics using the analytical equations for an ideal octupole field:

$$
\begin{aligned}
& H_{x}=G\left(y^{3}-3 x^{2} y\right) \\
& H_{y}=G\left(3 x y^{2}-x^{3}\right)
\end{aligned}
$$

where $G=B / a^{3}, B$ pole tip field, $a$ aperture radius.

The density contours at the target position are shown in Fig. 6. The achieved level of uniformity is not ideal. Nevertheless, it allows an increase of beam intensity by at least a factor of two if we assume a Gaussian input profile truncated at $2 \sigma$.

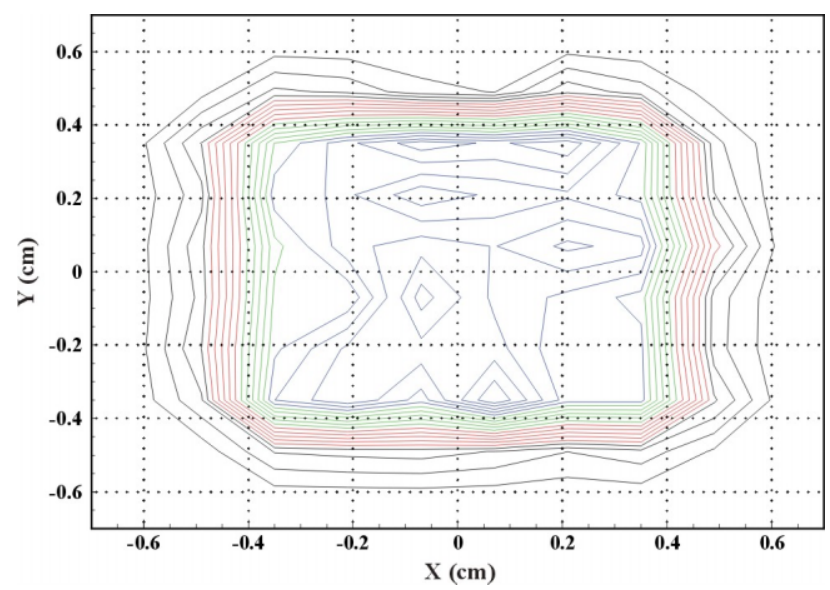

Fig. 6 Particle density contours at target position achieved by two octupoles.

\section{CONCLUSION}

DYNAMION is a versatile multi-particle code for simulations of transport and acceleration of charged particles. The general equations of motion are solved for realistic electromagnetic external fields presented by analytical formula, an internal Laplace solver, field maps or field measurements. Space charge is calculated by particle-particle interaction with high accuracy. DYNAMION has been successfully used for the design and evaluation of commissioning measurements at the upgraded UNILAC $[3,4]$.

\section{REFERENCES}

[1] U. Ratzinger, The New GSI Prestripper Linac for High Current Heavy Ion Beams, Proc. of LINAC96, Geneva, 1996

[2] A. Kolomiets, I. Vorobyov, S. Iaramishev (ITEP, Moscow, Russia), J. Klabunde (GSI, Darmstadt, Germany), DYNAMION - The Code for Beam Dynamics Simulations in High Current Ion Linacs, Proc. of EPAC98, Stockholm, 1998

[3] W. Barth, Commissioning of the $1.4 \mathrm{MeV} / \mathrm{u}$ High Current Heavy Ion Linac at GSI, Proc. of LINAC2000, Monterey, 2000

[4] W. Barth, L. Dahl, P. Forck, J. Glatz, J. Klabunde, Beam Measurements at the GSI High Current Injector, These Proceedings 